\title{
Ion Beam Analysis of Hydrogen-Treated Ti/TiN Protective Nanomultilayers
}

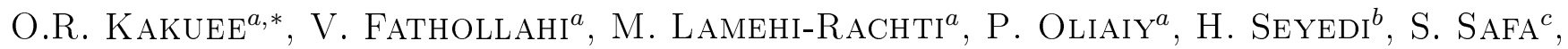 \\ M. Mojtahedzadeh LaRijani ${ }^{b}$ And H. Moazzami ${ }^{a}$ \\ ${ }^{a}$ Nuclear Science Research School, NSTRI, P.O. Box 14395-836, Tehran, Iran \\ ${ }^{b}$ Materials Research School, NSTRI, P.O. Box 31485-498, Karaj, Iran \\ ${ }^{c}$ Department of Nanotechnology, Faculty of Engineering, Tarbiat Modares University \\ P.O. Box 14115-143, Tehran, Iran
}

(Received September 10, 2011; in final form February 12, 2012)

\begin{abstract}
$\mathrm{Ti} / \mathrm{TiN}$ multilayer films with a few multilayer periods and a total sub- $\mu$ m thickness were deposited on AISI 304 stainless steel substrates by ion coating deposition technique. To investigate the effect of hydrogen treatment on the corrosion behavior of the multilayers, some of the samples were hydrogen treated after deposition of the first and/or the second Ti interlayer. ${ }^{14} \mathrm{~N}\left(\mathrm{~d}, \alpha_{1}\right){ }^{12} \mathrm{C}$ nuclear reaction and the Rutherford backscattering spectrometry were used to obtain the atomic composition profiles and thickness of $\mathrm{Ti} / \mathrm{TiN}$ layers. Nuclear reaction analysis confirmed the presence of two separable TiN layers of comparable thickness on the surface and in depth of the two-period multilayers. These techniques were used to determine the thickness of individual $\mathrm{Ti}$ and TiN layers and revealed that the stoichiometry of TiN layers was approximately $\mathrm{Ti}: \mathrm{N}=1: 1$. Hydrogen depth profile in the prepared samples was obtained by elastic recoil detection analysis. It was found that a remarkable volume of hydrogen was uptaken by the Ti layer in the hydrogen treated samples. The TiN (200) diffraction peak in the X-ray diffraction pattern was observed with different intensities depending on the sample preparation parameters. The corrosion behavior of the multilayers was studied by means of potentiodynamic polarization in $0.5 \mathrm{M} \mathrm{NaCl}$ solutions. It was found that the hydrogen treatment of $\mathrm{Ti}$ interlayer could potentially improve the corrosion properties of the $\mathrm{Ti} / \mathrm{TiN}$ layers.
\end{abstract}

PACS: 68.43.-h, 68.49.-h, 81.15.Jj, 25.45.-z

\section{Introduction}

TiN coatings are used for protection purposes on different kinds of substrate materials due to their hardness and corrosion resistance $[1,2]$. However, the corrosion resistance of monolayer physical vapour deposited (PVD) TiN films is poor due to imperfections within the layer such as microcracks, pores, and pinholes and the columnar microstructure of the coating [3].

To improve TiN coating performance, deposition of a thin Ti interlayer between the TiN film and the substrate was proposed as a transition layer which improves the adhesion strength, relieves the interfacial residual stress and blocks the pinholes in the TiN coating. In this way, the $\mathrm{Ti}$ interlayer prevents the approach of the corrosive medium to the substrate metal $[4,5]$.

Moreover, Ti/TiN multilayer coatings have been found to improve the hardness-, toughness-, and wear properties as well as the adhesion strength and the corrosion resistance of the metal substrate $[6,7]$.

Many research groups have been challenged to improve the mechanical and electrochemical properties of $\mathrm{Ti} / \mathrm{TiN}$ multilayers. Their efforts were associated with: (1) the preparation process of the multilayers including the $\mathrm{Ti}$ interlayer thickness, modulation period size and number

\footnotetext{
* corresponding author; e-mail: okakuee@yahoo.com
}

of cermet layers $[3-5,8,9]$ or the application of a suitable deposition technique and optimization of the deposition parameters [10-12]; (2) the application of post-coating treatments such as in-air thermal oxidation of the multilayer [13] or annealing the TiN layer in vacuum at various temperatures [14].

The beneficial effect of annealing in hydrogen atmosphere on the corrosion resistance of certain alloys has already been reported [15]. It has also been shown that hydrogen could control the microstructure of titanium alloys and thereby improve their physical properties [16]. Moreover, as pointed out earlier, the formation of $\mathrm{Ti}$ interlayers is necessary for the preparation of $\mathrm{Ti} / \mathrm{TiN}$ multilayers. With regard to these facts, the idea of hydrogen treatment of $\mathrm{Ti}$ interlayers was investigated as an alternative approach for improving the corrosion behavior of $\mathrm{Ti} / \mathrm{TiN}$ multilayers in this work.

To conduct this research work, samples of Ti and TiN layers, each of them tens of nanometers thick were deposited on stainless steel substrates by PVD technique. To evaluate the effect of hydrogen treatment on corrosion resistance of substrate, the Ti interlayers were hydrogen treated under specific experimental conditions.

The phase composition of the coatings was investigated by X-ray diffraction (XRD). Ion beam analysis of the samples was carried out using the $3 \mathrm{MV}$ Van de Graaff accelerator of NSTRI/Tehran. Information on the elemental distribution and thickness of the coatings 
was obtained by Rutherford backscattering spectrometry (RBS). Elastic recoil detection analysis (ERDA) was employed to analyze hydrogen in the prepared samples. To determine the presence of nitrogen in the prepared samples, nuclear reaction analysis (NRA) was used.

To reveal the effect of hydrogen treatment of $\mathrm{Ti}$ inter-layers on the corrosion resistance of the prepared samples, potentiodynamic polarization measurements were carried out in $0.5 \mathrm{M} \mathrm{NaCl}$ solution.

\section{Experimental}

\subsection{Sample preparation}

The $0.9 \mathrm{~mm}$ thick AISI 304 stainless steel plate was cut into square pieces of $12 \mathrm{~mm}$ sides. The substrates were then cleaned using ultrasonic cleaner; first in acetone, and then in ethanol before introduction to the ion coating chamber where the substrate surface was cleaned for the last time by argon ion bombardment at a negative substrate bias of $800 \mathrm{~V}$ and at pressure of $6.7 \mathrm{~Pa}$ for $20 \mathrm{~min}$.
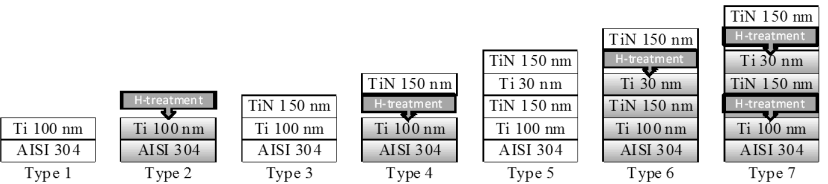

Fig. 1. Arrangement and thickness of constituting layers in 7 different types of prepared samples; shaded areas indicate that the prepared layers were hydrogen treated.

In this investigation, seven different types of samples were prepared as schematically shown in Fig. 1. In the same figure, the thickness of each layer is also illustrated.

\subsubsection{Deposition of Ti layer}

Coating material in the form of granular titanium which was charged in the graphite crucible of the ion coating system was evaporated and ionized by a hollow cathode discharge gun as the evaporation source. The electron current incident on the charge material by the electron gun was 120 A. The ionized material was directed towards the substrate under a bias voltage of $60 \mathrm{~V}$ and deposited on the substrates pre-heated at $380^{\circ} \mathrm{C}$.

\subsubsection{Deposition of TiN layer}

For the deposition of TiN layers, nitrogen was introduced in the chamber of the system and TiN was reactively formed and deposited on the substrates at a bias voltage of $60 \mathrm{~V}$. The base pressure of the system was $4.3 \times 10^{-2} \mathrm{~Pa}$, while the working pressure was kept at $5.9 \times 10^{-2} \mathrm{~Pa}$. The electron current incident on the charge material was $120 \mathrm{~A}$ while the substrates were preheated at $380^{\circ} \mathrm{C}$.

\subsubsection{Hydrogen treatment of samples}

For the hydrogen treatment, samples were primarily outgassed in a vacuum of $10^{-6}$ mbar at $300^{\circ} \mathrm{C}$ for $2 \mathrm{~h}$. Hydrogen uptake was then carried out at ambient temperature under the hydrogen pressure of 5 atm for $24 \mathrm{~h}$.
Details of the hydrogen treatment and the corresponding experimental setup have previously been published by the authors [17].

\subsection{Corrosion tests}

The corrosion resistance of the multilayers was evaluated using potential-current curves obtained by potentiodynamic polarization scans in $0.5 \mathrm{M} \mathrm{NaCl}$ solution at $20^{\circ} \mathrm{C}$. A Cu wire was soldered to the uncoated side of the sample plate. The uncoated surfaces and edges were sealed in epoxy resin, exposing a surface area of $1 \mathrm{~cm}^{2}$ to the test solution as the working electrode. After cleaning the samples in ultrasonic bath (acetone, $15 \mathrm{~min}$ ), all the electrode potentials were measured against $\mathrm{Ag} / \mathrm{AgCl}$ reference electrode. A classical three-electrode cell configuration was used (Metrohm 797 Computrace). The counter electrode was a $\mathrm{Pt}$ foil $\left(1 \mathrm{~cm}^{2}\right)$ and the potentiodynamic polarization curves were obtained at a scan rate of $0.15 \mathrm{mV} / \mathrm{s}$ in the potential range of OCP $500 \mathrm{mV}$ to $600 \mathrm{mV}$. The corrosion current density was estimated by linear fit and Taffel extrapolation to the cathodic part of the polarization curve.

\subsection{Coating characterization}

To obtain the atomic composition profiles and the thickness of Ti/TiN layers, NRA and RBS were employed using a single ended $3 \mathrm{MV}$ Van de Graaff accelerator. For NRA analysis, the ${ }^{14} \mathrm{~N}\left(\mathrm{~d}, \alpha_{1}\right){ }^{12} \mathrm{C}$ reaction with the well-known cross-sections was considered and a $1400 \mathrm{keV}$ deuterium beam was used to bombard the samples. In this way, the distribution profile of $\mathrm{N}$ in the TiN layers was determined. For the RBS analysis, a $2200 \mathrm{keV}$ alpha beam was incident on the sample. A surface barrier detector, $300 \mu \mathrm{m}$ thick, equipped with standard electronics set at $165^{\circ}$ was used to measure the energy of backscattered particles in both RBS and NRA analyses.

\subsection{Hydrogen depth profiling}

In order to determine the hydrogen depth profile in the $\mathrm{Ti}$ /TiN multilayer, ERDA was applied. In this analysis, the samples were irradiated with an $1800 \mathrm{keV}^{4} \mathrm{He}^{+}$beam of $1 \mathrm{~mm}$ diameter generated by the Van de Graaff accelerator. The incident angle of the beam was $80^{\circ}$. The hydrogen atoms recoiled in the forward direction were detected with a surface barrier detector at an angle of $25^{\circ}$ to the beam direction. A $7 \mu \mathrm{m}$ thick kapton foil was placed in front of the detector to filter out the incident ions scattered by the sample.

\section{Results and discussion}

Figure 2 shows the experimental backscattering spectra obtained by the $2200 \mathrm{keV}$ alpha-particle bombardment of the type 2 , type 4 and type 5 samples. The peaks in these spectra correspond to the Ti layers and the plateaus in the higher energy side of $\mathrm{Ti}$ peaks in the spectra of samples from type 4 and type 5 to the TiN layers. The thickness of individual layers of $\mathrm{Ti}$ and $\mathrm{TiN}$ were 
determined by simulation of the RBS spectra using the SIMNRA software [18]. The thickness of the individual layers is indicated in Fig. 1.

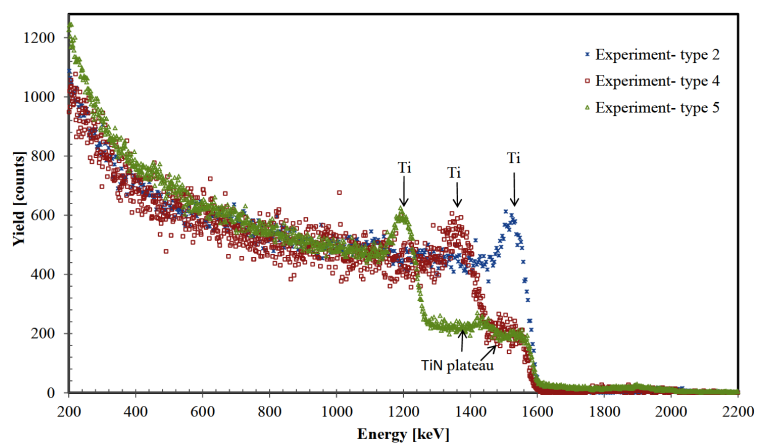

Fig. 2. The experimental RBS spectra for a $2200 \mathrm{keV}$ alpha beam incident on samples of type 2 (a single Ti layer), type 4 (a pair of $\mathrm{Ti} / \mathrm{TiN}$ layers) and type 5 (two pairs of $\mathrm{Ti} / \mathrm{TiN}$ layers).

Figure 3 shows the NRA spectra obtained by the deuteron bombardment of the type 4 (a pair of $\mathrm{Ti} / \mathrm{TiN}$ layers) and type 5 (two pairs of Ti/TiN layers) samples $\left({ }^{14} \mathrm{~N}\left(\mathrm{~d}, \alpha_{1}\right){ }^{12} \mathrm{C}\right.$ nuclear reaction $)$. The NRA spectrum of the type 4 sample (a pair of $\mathrm{Ti} / \mathrm{TiN}$ layers) shows a single peak of nitrogen originating from the single TiN layer. On the other hand, the two distinct peaks of comparable intensity in the NRA spectrum of the type 5 sample (two pairs of $\mathrm{Ti} / \mathrm{TiN}$ layers) indicate the presence of two separable TiN layers of comparable thickness on the surface and in depth of the sample.

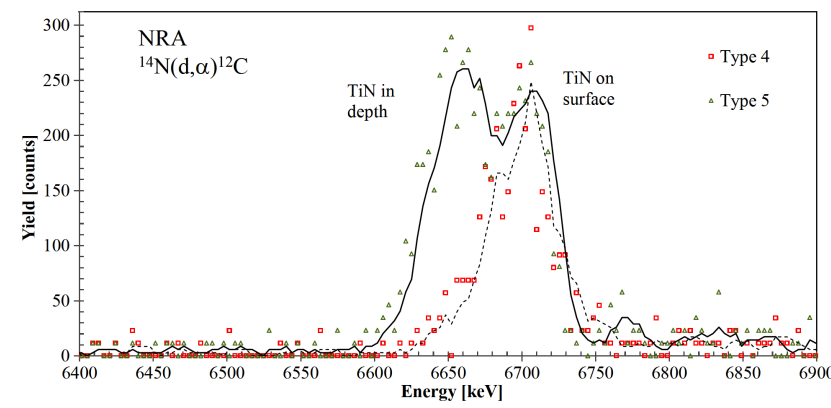

Fig. 3. The ${ }^{14} \mathrm{~N}\left(\mathrm{~d}, \alpha_{1}\right){ }^{12} \mathrm{C}$ NRA spectra of samples of type 4 (a pair of $\mathrm{Ti} / \mathrm{TiN}$ layers) and type 5 (two pairs of $\mathrm{Ti} / \mathrm{TiN}$ layers).

Figure 4 shows the ERDA hydrogen spectra of the type 1 (a single Ti layer with no hydrogen treatment) and type 2 (a single Ti layer which is hydrogen treated) samples along with the corresponding spectra simulated by SIMNRA.

As it could be expected, the hydrogen concentration in the samples not treated with hydrogen (type 1 sample), is low and very likely originating from the adsorption of ambient hydrogen by the $\mathrm{Ti}$ layer. On the other hand, a higher amount of hydrogen can be observed in the sur-

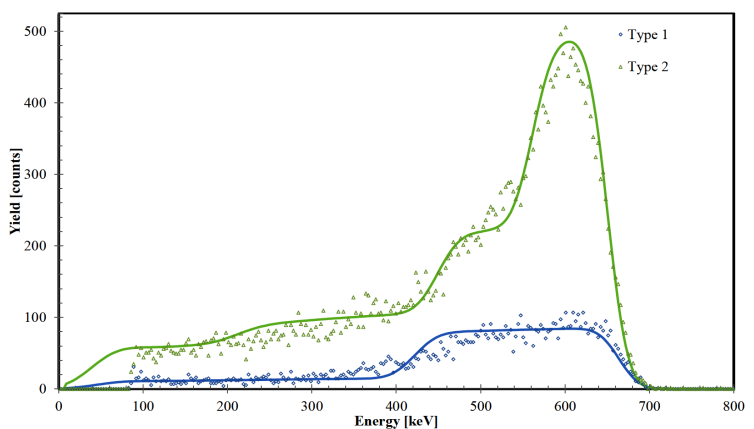

Fig. 4. The ERDA experimental data and corresponding SIMNRA simulated curves for the pristine single $\mathrm{Ti}$ layer (sample of type 1) and hydrogen-treated single $\mathrm{Ti}$ layer (sample of type 2) $\left(E_{\alpha}=1800 \mathrm{keV}, \alpha=80^{\circ}\right.$, $\beta=75^{\circ}$ and $\theta=25^{\circ}$; stopping foil: $7 \mu \mathrm{m}$ thick kapton).

face layer of hydrogen-treated sample (type 2 sample). According to the SIMNRA fit, the concentrations of hydrogen in the $\mathrm{Ti}$ coated layer (corresponding to the peak in ERDA spectra) for the type 1 and 2 samples were 2.8 and 11 at.\%, respectively. While across the low-energy plateau of this region, the hydrogen concentrations varied to 0.6 at. $\%$ and 2.9 at.\% for the samples of type 1 and 2, respectively. Therefore, it can be concluded that the adopted hydrogen treatment was effective and a considerable amount of hydrogen was bound by the Ti coated layer.

Furthermore, the obtained values for the adsorbed hydrogen in the type 1 and type 2 samples indicate that:

a) hydrogen is present even in pristine (with no hydrogen treatment) AISI 304 stainless steel substrate;

b) considerable amount of hydrogen could be adsorbed by the AISI 304 stainless steel substrate during hydrogen treatment.

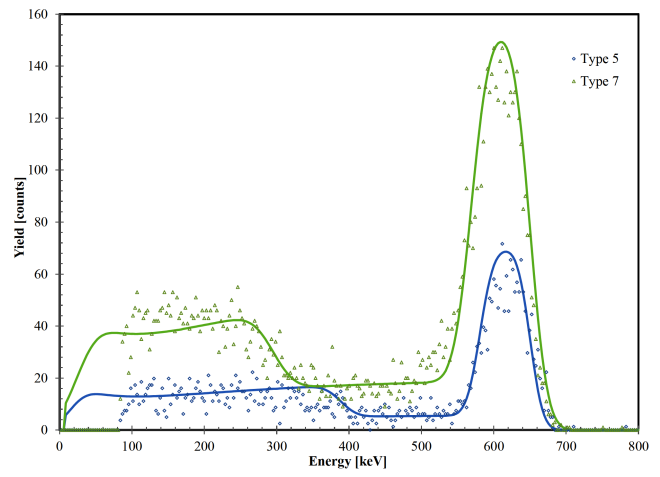

Fig. 5. The ERDA experimental data and corresponding SIMNRA simulated curves for the Ti/TiN multilayers of type 5 and type 7 samples $\left(E_{\alpha}=1800 \mathrm{keV}\right.$, $\alpha=80^{\circ}, \beta=75^{\circ}$ and $\theta=25^{\circ}$; stopping foil: $7 \mu \mathrm{m}$ thick kapton).

Figure 5 presents the ERDA hydrogen spectra of the type 5 (two pairs of $\mathrm{Ti} / \mathrm{TiN}$ layers with no hydrogen 
treatment) and type 7 (two pairs of $\mathrm{Ti} / \mathrm{TiN}$ layers that are hydrogen treated) samples along with the SIMNRA simulated curves for the corresponding spectra. On each ERDA spectrum, three distinct areas could be identified: a surface peak corresponding to the fourth coated layer, a valley corresponding to the penultimate $\mathrm{Ti}$ layer and a low-intensity plateau corresponding to in-depth TiN layer. The hydrogen concentration in each of these areas for samples of type 5 and 7 are summarized in Table I. It should be noted that the underlying TiN layer cannot be explored by ERDA with the selected experimental setup and beam energy. For this reason the concentration of hydrogen in this layer is not reported.

Obviously, the intensity of hydrogen in the ERDA spectrum of the pristine type 5 sample (sample with no hydrogen treatment) is low which could be assigned to the adsorption of ambient hydrogen by the $\mathrm{Ti} / \mathrm{TiN}$ layers.

With regard to the results of RBS, NRA and ERDA analyses summarized in Table I, the SIMNRA simulation proposes a stoichiometry of $c a .50$ at. $\%$ Ti and 50 at.\% N for the TiN layers, while the hydrogen content of the samples varies from 0.5 at. $\%$ to 3.0 at.\% depending on whether the sample was hydrogen-treated or not.

In Table I, it is evident that TiN layers are composed of approximately 50 at.\% Ti and 50 at.\% $\mathrm{N}$ and a varying hydrogen from 0.7 at.\% to 2.9 at.\%. However, the nitrogen content of Ti layers varies from 16.6 at.\% to 30 at.\%, while the hydrogen content of the same layers varies from 0.4 at.\% to 1.4 at.\%.

TABLE I

Hydrogen, nitrogen and titanium concentration (at.\%) of each coated layer obtained from SIMNRA simulation of ion beam analysis measured spectra from sample types $3,4,5$ and 7 .

\begin{tabular}{c|c|c|c|c|c|c|c|c|c|c|c|c}
\hline \hline & \multicolumn{3}{|c|}{$\begin{array}{c}\text { First coating layer } \\
(\mathrm{Ti})\end{array}$} & \multicolumn{3}{c|}{$\begin{array}{c}\text { Second coating layer } \\
(\mathrm{TiN})\end{array}$} & \multicolumn{2}{c|}{$\begin{array}{c}\text { Third coating layer } \\
(\mathrm{Ti})\end{array}$} & \multicolumn{3}{c}{$\begin{array}{c}\text { Fourth coating layer } \\
(\mathrm{TiN})\end{array}$} \\
\cline { 2 - 13 } & $\mathrm{Ti}$ & $\mathrm{N}$ & $\mathrm{H}$ & $\mathrm{Ti}$ & $\mathrm{N}$ & $\mathrm{H}$ & $\mathrm{Ti}$ & $\mathrm{N}$ & $\mathrm{H}$ & $\mathrm{Ti}$ & $\mathrm{N}$ & $\mathrm{H}$ \\
\hline type 3 & 73.8 & 25 & 1.2 & 49.2 & 48 & 2.8 & & & & & & \\
type 4 & 82 & 16.6 & 1.4 & 50.1 & 47 & 2.9 & & & & & & \\
type 5 & - & - & - & 51.2 & 48.1 & 0.7 & 69.6 & 30 & 0.4 & 49.3 & 49.2 & 1.5 \\
type 7 & - & - & - & 49 & 49.5 & 1.5 & 74.2 & 25 & 0.8 & 47 & 50.2 & 2.8
\end{tabular}

It should be noted that the higher values of hydrogen in the uppermost layers could originate from adsorption of atmospheric hydrogen by the Ti atoms. Moreover, for interpretation of the hydrogen content of different layers in Table I, one should consider the fact that hydrogen could be redistributed in the in-depth layers due to preheating of the samples during the hydrogen treatment or layer deposition. One more point which should be considered for understanding the measured hydrogen content data in different layers, is that TiN layers could block hydrogen diffusion because they have a low hydrogen permeability coefficient [19]. Therefore, higher hydrogen content in the TiN layers could be justified. At the same time, one should keep in mind that preheating of the samples during hydrogen treatment and fabrication of successive layers could stimulate the outwards diffusion of hydrogen atoms and hence a lower hydrogen concentration could be observed in samples with hydrogen treated $\mathrm{Ti}$ interlayers in comparison with the single hydrogen treated $\mathrm{Ti}$ layer.

Figure 6 shows the X-ray diffraction patterns of the seven types of the prepared samples. These data were recorded by means of a PW1800 Philips diffractometer using the $\mathrm{Cu} K_{\alpha}$ radiation. In all XRD patterns the presence of the diffraction peaks at $43.4^{\circ}, 50.6^{\circ}, 74.6^{\circ}$ and $90.5^{\circ}$ associated with the AISI 304 stainless steel substrates could be observed.

In the XRD patterns of the samples of type 3 onwards one could observe the development of a rather broad peak

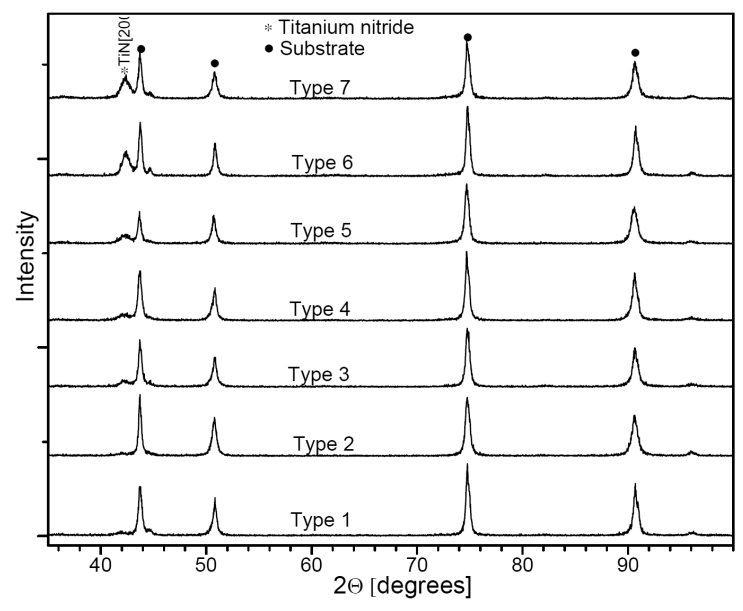

Fig. 6. X-ray diffraction patterns of prepared samples of different types.

at a $2 \theta$ angle smaller than those of the stainless steel substrate. This peak which corresponds to diffraction from (200) planes of titanium nitride, is strongly developed in type 5, 6 and 7 samples where the arrangement and number of constituting layers are identical. However, the intensity of (200) titanium nitride peak is higher for samples of the type 6 and 7 which were subjected to hydrogen treatment once or twice. Moreover, in samples of the types 6 and 7 , we observe another peak with low inten- 
sity at about $44^{\circ}$ corresponding to $\mathrm{TiH}_{2}(002)$ phase. The High intensity of TiN peak adjacent to the $\mathrm{TiH}_{2}$ phase in these samples is due to a decrease of lattice mismatch (about 1\%) between the two phases in comparison with that of other samples and probably originates from the microstructure of TiN sublayer.

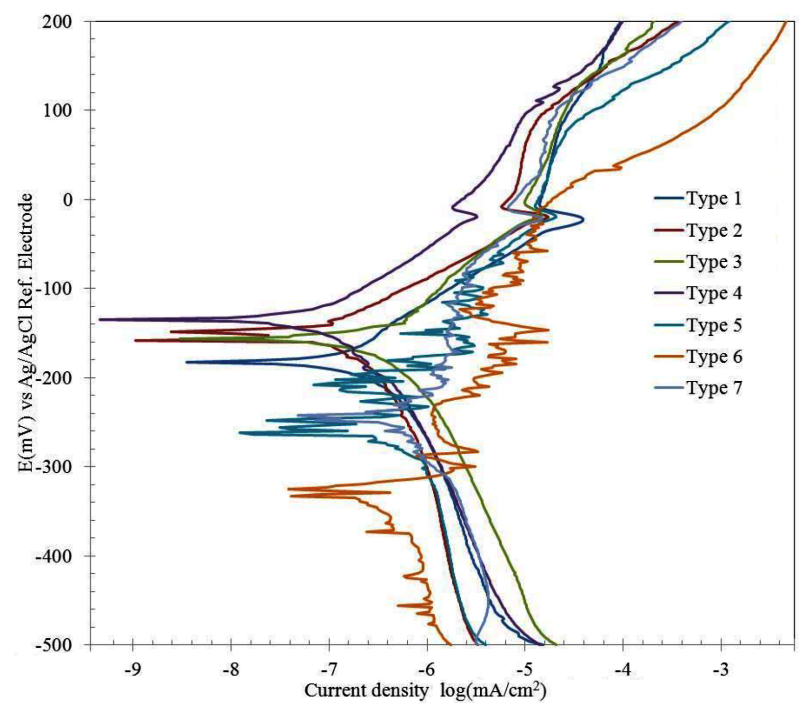

Fig. 7. Polarization curves of the prepared samples of different types in $0.5 \mathrm{M} \mathrm{NaCl}$ solution.

The potentiodynamic polarization curves of the prepared samples of different types in $0.5 \mathrm{M} \mathrm{NaCl}$ solution are shown in Fig. 7. The most important electrochemical parameters of the samples i.e. $E_{\text {corr }}$ and $I_{\text {corr }}$ are summarized in Table II.

\section{TABLE II}

Some electrochemical parameters for the different types of prepared samples.

\begin{tabular}{c|c|c}
\hline \hline Sample & $E_{\text {corr }}[\mathrm{mV}]$ & $I_{\text {corr }}\left[\mu \mathrm{A} / \mathrm{cm}^{2}\right]$ \\
\hline type 1 & -180 & $1.0 \times 10^{-1}$ \\
type 2 & -145 & $8.3 \times 10^{-2}$ \\
type 3 & -159 & $1.26 \times 10^{-1}$ \\
type 4 & -132 & $4.3 \times 10^{-2}$ \\
type 5 & -250 & $3.2 \times 10^{-1}$ \\
type 6 & -330 & $3.2 \times 10^{-1}$ \\
type 7 & -253 & $1.74 \times 10^{-1}$
\end{tabular}

By comparing $E_{\text {corr }}$ and $i_{\text {corr }}$ of the type 1 and 2 samples, one can deduce that the hydrogen treatment has improved the corrosion resistance properties of the samples.

The type 4 sample, where a TiN layer was deposited on an $H$-treated Ti layer, shows improved $E_{\text {corr }}$ and $i_{\text {corr }}$ values in comparison with those of the type 3 sample, which was not subjected to $H$-treatment.

It seems that hydrogen treatment has positively affected the corrosion resistance of the samples. This could be explained by improvement of Ti layer microstructure due to hydrogen treatment which has already been reported by Goltsov [16].

For samples of the types 6 and 7 the $E_{\text {corr }}$ and $i_{\text {corr }}$ have poor values which could be due to formation of blisters originating from diffusion of hydrogen, while the samples were heated during hydrogen treatment. In this process, combination of atomic hydrogen in coated layers could lead to the formation of molecular hydrogen. Hydrogen blisters are normally developed when these molecular hydrogen are accumulated in voids and other defect sites. In fact, hydrogen blistering is one of the main hydrogen damage mechanisms observed in metallic corrosion [20].

\section{Conclusion}

Samples of Ti/TiN multilayers deposited on 304 stainless steel and hydrogen treated were characterized by IBA techniques. Thicknesses of individual $\mathrm{Ti}$ and TiN layers, stoichiometry of the TiN layers, as well as depth profile of hydrogen and nitrogen were determined by RBS, NRA, and ERDA.

The results of our investigation showed that hydrogen treatment of $\mathrm{Ti}$ coated layer positively affects the corrosion resistance of samples with single $\mathrm{Ti}$ coated layer, as well as samples with single-period $\mathrm{Ti} / \mathrm{TiN}$ layers. One could expect that samples protected by two-period $\mathrm{Ti} / \mathrm{TiN}$ multilayer would show high corrosion resistance. However, it seems that heating the samples during TiN deposition and hydrogen treatment could lead to hydrogen diffusion and formation of hydrogen blisters which in turn deteriorates corrosion resistance of the samples. Moreover, the measured hydrogen contents in different layers support the fact that TiN coatings could block hydrogen diffusion due to their low hydrogen permeability coefficient. It was also found that formation of $\mathrm{TiH}_{2}$ phase could make an interface with lower lattice mismatch for the growth of $\operatorname{TiN}(200)$ phase.

\section{References}

[1] R. Morita, K. Azuma, S. Inoue, R. Miyano, H. Takikawa, A. Kobayashi, E. Fujiwara, H. Uchida, M. Yatsuzuka, Surf. Coat. Technol. 136, 207 (2001).

[2] I. Milosev, H.-H. Strehbtow, B. Navinsek, Thin Solid Films 303, 246 (1997).

[3] M. Flores, S. Muhl, L. Huerta, E. Andrade, Surf. Coat. Technol. 200, 1315 (2005).

[4] J.-H. Huang, F.-Y. Ouyang, G.-P. Yu, Surf. Coat. Technol. 201, 7043 (2007).

[5] J.-H. Huang, C.-H. Ma, H. Chen, Surf. Coat. Technol. 200, 5937 (2006).

[6] C. Liu, G. Lin, D. Yang, M. Qi, Surf. Coat. Technol. 200, 4011 (2006).

[7] C. Liu, P.K. Chu, G. Lin, D. Yang, Corrosion Sci. 49, 3783 (2007).

[8] Q. Zhang, Y.X. Leng, F. Qi, T. Tao, N. Huang, Nucl. Instrum. Methods Phys. Res. B 257, 411 (2007). 
[9] Y.H. Cheng, T. Browne, B. Heckerman, C. Bowman V. Gorokhovsky, E.I. Meletis, Surf. Coat. Technol. 205, 146 (2010).

[10] C.Z. Chen, Q. Lia, Y.X. Leng, J.Y. Chen, P.C. Zhang, B. Bai, N. Huang, Surf. Coat. Technol. 204, 3082 (2010).

[11] M. Flores, S. Muhl, E. Andrade, Thin Solid Films 433, 217 (2003).

[12] M. Flores, L. Huerta, R. Escamilla, E. Andrade, S. Muhl, Appl. Surf. Sci. 253, 7192 (2007).

[13] E. Galvanetto, F. Borgioli, F.P. Galliano, T. Bacci, Surf. Coat. Technol. 200, 3650 (2006).

[14] R Y. Xi, H. Fan, W. Liu, J. Alloys Comp. 496, 695 (2010).

[15] G.J. Liu, S.S. Jia, Y.F. Zhu, S.H. Hong, J.W. Lim, K. Mimura, M. Isshiki, Mater. Sci. Eng. A 472, 235 (2008).
[16] V.A. Goltsov, Int. J. Hydrogen Energy 27, 845 (2002).

[17] S. Safa, M. Mojtahedzadeh Larijani, V. Fathollahi, O.R. Kakuee, Nano 5, 341 (2010).

[18] M. Mayer, SIMNRA User's Guide, Report IPP 9/113, Max-Planck-Institut für Plasmaphysik, Garching, Germany 1997.

[19] K. Saito, S. Inayoshi, Y. Ikeda, Y. Yang, S. Tsukahara, J. Vac. Sci. Technol. A 13, 556 (1995).

[20] P.A. Schweitzer, Fundamentals of Corrosion: Mechanisms, Causes and Preventative Methods, CRC Press, New York 2010 